\title{
Fleet Management and Grid Integration of PV Generating Stations
}

\author{
Kiriakos Siderakis $^{1}$, Antiopi Gigantidou ${ }^{2}$, Konstantinos Lagouvardos ${ }^{3}$, Eleftheria Kavousanaki ${ }^{1}$,
}

Fotis Mavromatakis $^{1}$ and Dimitrios Kounalakis ${ }^{1}$

\begin{abstract}
The integration of renewable sources in electric power systems motivates considerable changes, to many aspects related with their operation and control. The rate of change is further accelerated in the case of Photovoltaic Generation $(P V)$ due to features that this technology demonstrates. The impacts that $P V$ generation can have, in the power grid operation, are investigated in this paper. Further, considering these impacts, management schemes that will allow the operator to assume the operation of a virtual $P V$ generator, containing a fleet of $P V$ stations are investigated. The analysis relies to the power system of Crete, which is a very interesting case study, considering the production scheme and the integration of RES.
\end{abstract}

\section{Keywords}

Fleet management, grid integration, photovoltaic generation, isolated power systems

\section{Acknowledgement}

This project is implemented through the Operational Program "Education and Lifelong Learning", Action Archimedes III and is co-financed by the European Union

\footnotetext{
${ }^{1}$ Laboratory of Energy and Photovoltaic Systems, Department of Electrical Engineering, Technological Educational Institute of Crete, Greece, Stauromenos, GR71004, tel: +302810-379231, fax: 2810-379328

(e-mail: ksiderakis@ staff.teicrete.gr, fotis@ staff.teicrete.gr)

${ }^{2}$ Hellenic Electricity Distribution Network Operator, Terma Kastorias, Katsambas, Heraklion, Crete, GREECE, GR71307, tel: +302810-303218, fax: +302810-229762 (e-mail: a.gigantidou@deddie.gr)
}

\footnotetext{
${ }^{3}$ Institute for Environmental Research and Sustainable Development, National Observatory of Athens
}

(European Social Fund) and Greek national funds (National Strategic Reference Framework 2007 - 2013).

\section{Introduction}

The introduction of renewable sources (RES) in today's power systems initiates a fundamental change of the system operation. The new topology schemes and especially the change on the position of the generating nodes, in respect to the loads, can be quite changeling, both for the coordination of production and the transmission and distribution networks operation $[1,2]$. Considering in addition that during the last two decades the required supply quality levels have also been considerably increased, the problem of operating RES enhanced power systems have become quite complicated and the introduction of more efficient systems and techniques is necessary [3,4]. Such an increase of the system intelligence can be evident by the development of Smart Grids, which will be able to determine the operation of a power grid, incorporating both enhanced data collection and efficient control techniques [6, 7]. Furthermore, until the establishment of smart grids, a transition period is required, during which the integration of RES production is implemented in conventional systems and must be efficiently operated in order to satisfy the specifications and the quality and reliability levels required.

Furthermore, the problems recognized above, can be further enhanced as the size of the power system is decreased. In the case of small systems, the operation of RES and especially sources where the control ability is limited, can become critical, considering that the amount of RES generation can be comparable or even in excess of

This is an Open Access article distributed in accordance with the Creative Commons Attribution Non Commercial (CC-BY-NC-ND 4.0) license, which permits others to copy or share the article, provided original work is properly cited and that this is not done for commercial purposes. Users may not remix, transform, or build upon the material and may not distribute the modified material (http://creativecommons.org/licenses/by-nc/4.0/) 
the load requirements $[8-10]$. In such case the system stability and power supply quality can become critical issues. Therefore, the introduction of RES in small power systems, should comply with more tight restrictions and require coordinated actions.

\section{Forms of RES in respect to the operation of power systems}

Considering the levels of production, the first step towards RES oriented power systems, is the incorporation of hydro generation, is today the RES form of energy with the larger production levels and installed capacity [11]. In addition in this case, the supply of the primary source of energy can be controlled by the operator. Consequently, hydro generation is a form of RES that can be easily incorporated in conventional power systems. Unfortunately the primary source supply, illustrates limited availability and as a result hydro generation is possible only in specific geographical areas.

In the contrary, other forms of RES such as wind and solar power are available in wider geographical areas, around the world and therefore the exploitation of these forms is in the interest of more people. Unfortunately, these two illustrate two important drawbacks. Firstly, the availability of the primary energy source, i.e. the availability of wind and sun radiation cannot be controlled coordinate the energy production with the load demand. In addition, the energy production efficiency is low, especially in the case of solar energy. As a result, the point of installation is determined by the level of energy availability, regardless of the grid operation and in addition, in order to ensure notable levels of energy production, large scale installations are required [12]

Instead of that, the necessary levels of energy generation, can be achieved by forming fleets of generating stations, which can assemble a virtual power station, with installed capacity comparable to the capacity of conventional stations. This scheme, known as distributed generation, is quite promising, since it can efficiently support the integration of RES in power systems and optimize the energy generation, considering that the location of the RES station is not limited by the grid operation. However, as mentioned before, controlling of the generating fleet is a difficult issue, both due to the uncertainty of the primary source and the geographical distribution.

Further, when evaluating the wind and solar electricity integration, considerable differences can be found. Probably the most important difference is the levels of energy distribution per installation surface, which is in favor of wind generation. Photovoltaics have considerably lower levels, thus requiring larger installation areas than wind generation. This difference, combined with the capability of photovoltaics to be integrated in buildings, results in a PV generation scheme formed of small scale installations, capable of supplying power at the levels of kilowatts. Such installations are then connected to the low voltage distribution grid.

\section{The power system of Crete, an interesting case study}

The power system of Crete is an isolated power system with increased levels of RES penetration, including both wind and solar power. With an installed capacity of $850 \mathrm{MW}$ in three conventional power stations and a peak load exceeding $585 \mathrm{MW}$, the amount of wind generation installed capacity is $184 \mathrm{MW}$ and $116.2 \mathrm{MW}$ the PV generation installed capacity (figure 1). In figure 2 , the distribution of the generating stations in respect to the installed capacity is illustrated.

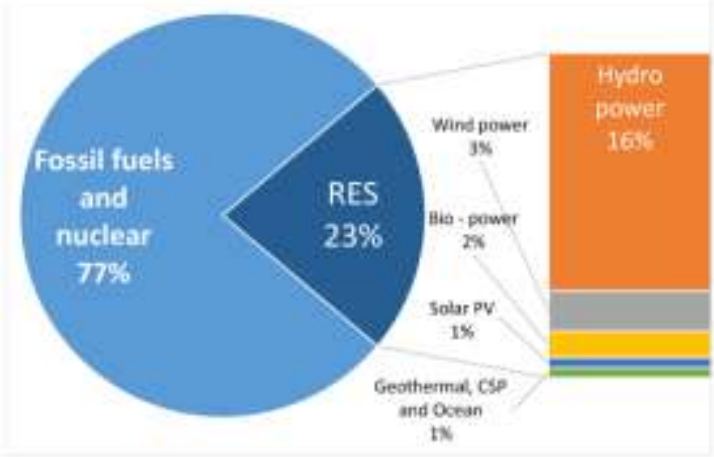

Fig. 1 Installed generating capacity of the technologies in use in the Power System of Crete

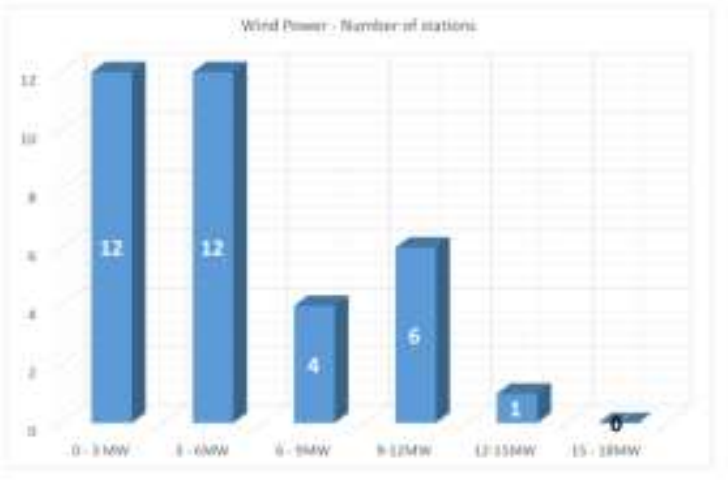




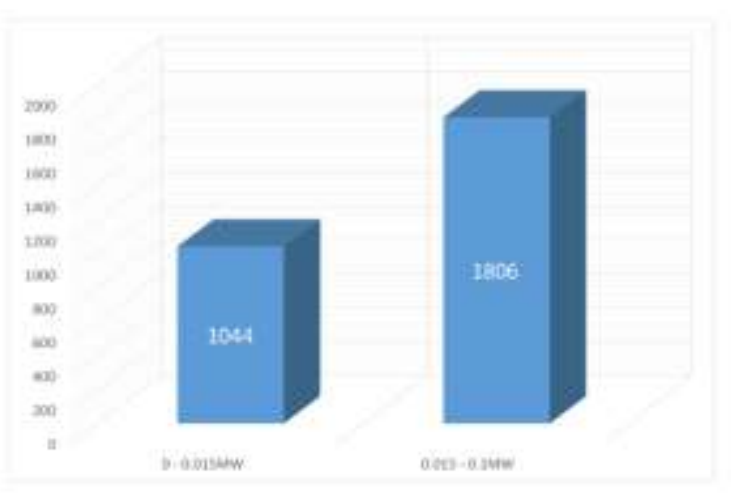

Fig. 2 Distribution of RES stations, according to their installed capacity the Power System of Crete

The difference of the unit installed capacity, between wind and PV generation is evident. The number of wind generating stations in Crete is 35 , where the average wind power installed capacity, per station is $5.84 \mathrm{MW}$, with the smaller station found at $0.5 \mathrm{MW}$ and the largest at 14.45MW. On the other hand, in the case of PV generation, $80 \mathrm{~kW}$ systems and $10 \mathrm{~kW}$ roof top systems are the majority of the installations found, forming a fleet of 1044 and 1806 units respectively. The large difference in the installed capacity results also to different connection schemes [13]. Wind stations are connected to the $20 \mathrm{kV}$ network, with dedicated distribution lines, thus directly to the $20 \mathrm{kV}$ bus of the corresponding $150 \mathrm{kV} / 20 \mathrm{kV}$ substations, as illustrated in figure 3 . On the other hand the PV stations are connected to the $400 \mathrm{~V}$ network or the $20 \mathrm{kV}$ network, but on commercial and not dedicated

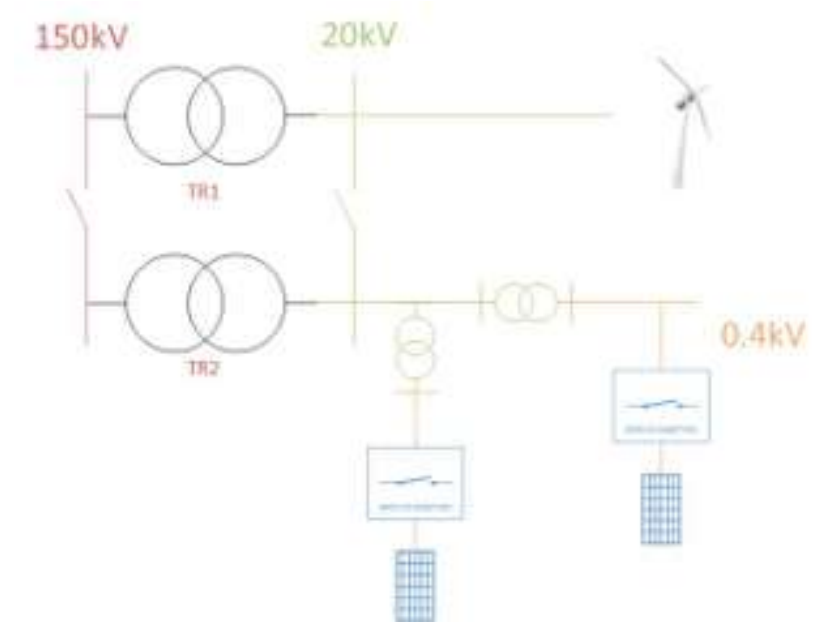

lines.

Fig. 3 Connection schemes of RES stations, in the Power System of Crete

\section{Possible impacts of PV generation in distribution networks}

The possible impacts of photovoltaic generation in distribution networks can be summarized as follows:

\subsection{Voltage variation}

(b) There are certain obligations that must be fulfilled regarding the voltage control, within the operation of $20 \mathrm{kV}$ and $0.4 \mathrm{kV}$ distribution networks. For the European countries for example, these obligations are included in EN60150 [14].

The problem that rises, due to the connection of PV stations in MV and LV networks, is the capability of the metwork operator to maintain the voltage, within the considered limits, along the grid lines $[15,16]$. In the scheme operating today, the voltage control relies on measurements at the MV bus of the HV/MV substation tap changer and the assumption that the voltage along a distribution line adapts a specific variation pattern [15]. The element implementing this control is the on load tap changer (OLTC), included in the HV/MV transformer.

Furthermore, considering the connection of PV stations along a distribution line, the voltage variation pattern may considerably change, since these stations supply energy near the loads and alter the energy flow that would be otherwise experienced. The distribution of the PV stations along the distribution line, in combination to the line load are key elements, in order to be able to operate efficiently the line.

In addition, when moving a step backwards and evaluating the operation of the OLTC, at the MV bus, it has to be noticed that the voltage controller of each OLTC operates on a number of transmission lines, assuming that all these lines share statistically common operational features. The penetration of PV generation may alter these common operational features and consequently the efficiency of the controller is decreased.

\subsection{Fault current levels contribution}

The presence of power generation in distribution lines also influences the anticipated fault current levels [18, 19]. This is an issue both for the endurance of the installed equipment, which has been selected according to certain fault current levels and the operation of the system protection systems. The exact implementation scheme must consider in addition, the operation of the PV inverters responsible for the grid connection. The settings and capabilities of these inverters are usually specified according to international standards and specifications set by the operators. 


\subsection{Current harmonics}

The operation of power electronics converters for the grid connection of PV stations is correlated also with the generation of current harmonics [19, 20]. The injection of these harmonics to the distribution grid may have impacts to the voltage quality and also initiate resonance phenomena in correlation to power factor correction capacitors.

\subsection{Substation power factor}

The contribution of PV generating stations to the distribution grid, primary concerns the supply of active power. Considering that the operation is implemented with a constant power factor, a reduction of the active power supplied from the high voltage grid, results to a substation power factor downgrade. This is an issue that may influence both the MV and HV grids, especially as the amount of PV penetration increases.

\subsection{Energy loss}

The energy loss, correlated with the operation of the distribution grid, can be decreased by the penetration of the PV generating stations [21, 22]. However it is important to consider that the degree of energy save achieved is correlated to the distribution of stations along a line. A typical scheme that illustrates the possible improvement, is the comparison in respect to the line losses of a PV Park connection with a dedicated line or on a typical distribution line. In the second case, energy efficiency is increased, since the amount of energy distributed from the high voltage substation is decreased.

\subsection{PV generation management at the system operator level}

Considering the operation of a conventional power system, the necessary equilibrium, between power generation and consumption, is managed by the main dispatch operator, which is responsible both for generation scheduling and real time management. From the dispatch operator point of view, PV generation management is difficult not only because the primary energy source cannot be controlled, but also because the installed capacity of each PV station is comparably small. In Crete for example, there are two levels at $80 \mathrm{~kW}$ and $10 \mathrm{~kW}$.

Furthermore, due to the small capacity, PV stations are integrated in the grid at the level of low or medium voltage. Thus, the possible influence to the high voltage grid, according to the parameters analyzed in the previous paragraph, is limited mainly to the total generation and substation power factor. The impact of the other parameters is limited to the medium and low voltage grids, due to the presence of the HV/MV transformers.

Consequently, for the main dispatch operator, estimating the total PV power generation is the primary concern, and especially the possible short term variation, since it is a requirement for efficient operation and generation scheduling. Further, in the case of an isolated system, in addition to the total generation, the PV generation for each HV/MV substation is also useful for the operation of the HV transmission system.

\section{Fleet management of PV generation}

Fleet management is a new approach within the operation of power systems, applied for the first time in PV generation [23, 24]. The need of such approach is evident both due to the size of the considered PV stations and their geographical distribution. The later may act in favour of the equivalent total PV generator, since the power output variation can be decreased. This is evident, as the degree of distribution increases, considering that the reasons of the power output fluctuations, vary in extent and time between stations.

An empirical model that illustrates this effect is illustrated by the correlation coefficient $\rho$, calculated in equation 1 [23]. The lower this correlation factor is the lower is the correlation between two stations and therefore a possible variation in the first station is not experienced simultaneously in the second station. Furthermore, for a fleet of stations, low values of this coefficient indicates that the total output variations may be smoothed due to the counterbalancing actions between stations.

$$
\rho=\frac{1}{1+\frac{\text { distance }}{(\Delta t)(\text { Cloud relative speed })}}
$$

A typical example is illustrated in figure 4 where the PV generation daily curves, for four stations in Crete are illustrated and the total curve is calculated, where the smoothing effect is evident. Further, as the number of stations considered is increased, the smoothing action is enhanced, and in a total the power generation curve is optimized. Consequently, fleet operation, where the installed power capacity is distributed in small generating units, geographically dispersed, results in an advantage as far as the power system operation is concerned. 


\section{The proposed generation management model}

The scope of the management model, is to be able to estimate the PV generation capability of a fleet of PV stations, exploiting available meteorological forecasts. The first step is predicting the PV generating capacity of a station, installed in a specific location. This model can be applied for all the stations connected to a HV/MV substation or to a number of selected stations. Then, the generating capacity of the fleet can be calculated, for each of the HV/MV substations and finally a total generating capability of the equivalent PV generator can be extracted.

In order to be able to estimate the daily PV generating capacity of a station, a model correlating the nominal power of the array under study, the temperature coefficient of the modules, the solar irradiance at the plane of the array, the air temperature and wind speed is implemented $[25,26]$. The results of the model for each station are then available to be grouped according to the power system structure and the calculation scope.

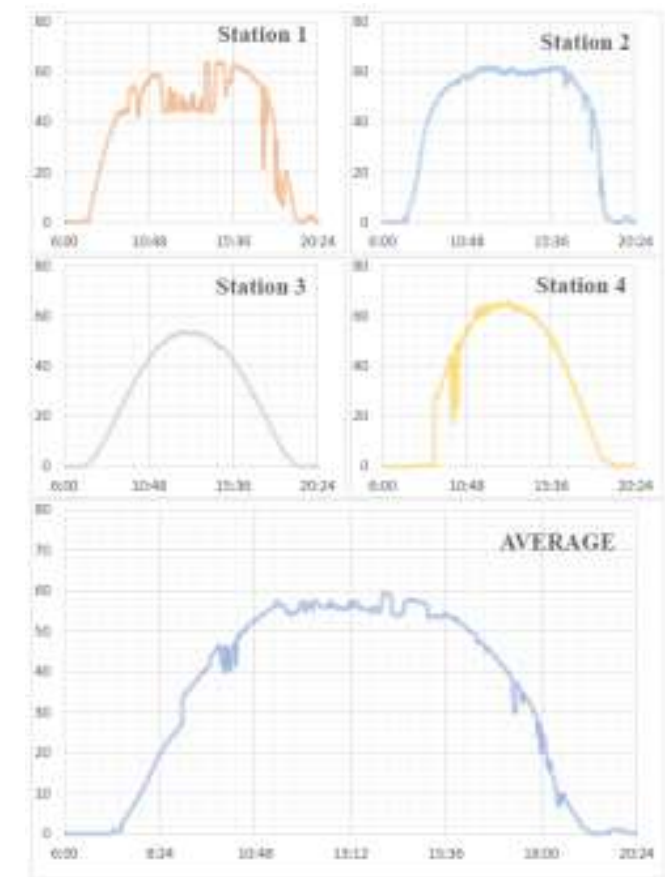

Fig. 4 Smoothing effect of PV stations production under the scheme of a virtual generator (power system of Crete)

An issue of investigation, is the methodology to be applied, in order to select the most representative group of stations, in case the total number of stations is large and thus computational heavy. The appropriate methodology must consider electrical and meteorological parameters in respect to the geographical location of each station. A possible implementation is to set the minimum number of stations to be considered (core stations). Then for each core station, a group will be formed, containing stations with correlation factors that exceed a user defined correlation factor value in respect to the core station. This model is similar to a cellular network, with the difference that the cell size is not constant but depends on parameters such as the stations distance from the core station, the speed of the clouds movement and the time interval of evaluation.

\section{Conclusions}

The integration of photovoltaic generation in power grids is a critical issue for the grid operators, since they bring a new aspect, in their operation and control. Due to reasons that are correlated both on the operation of the PV systems and the optimum integration in the power grids, the dispersion of the installed PV power capacity is new aspect for such systems.

Evaluating the operation of small scale PV stations, connected to the medium or low voltage grids, it can be concluded that there are impacts in the operation of these networks, which however have limited influences to the operation of the total system. In this case, fleets of PV stations can be formed, resulting to the concept of a virtual generator. The critical issue is the procedure to select the members of the fleet, in order to optimally demonstrate the PV generation. It can be seen that this procedure (fleet formation) is a dynamic procedure, which has to combine many parameters, such as the distance between stations, the clouds movement, the connection point etc.

Finally the basic scheme of a PV power prediction system is proposed, considering the operation of a PV generation station and the above mentioned parameters. This system incorporates a PV generation prediction model and a fleet management algorithm, capable of predicting the fore coming $\mathrm{PV}$ generation, in a power system.

\section{References}

[1] Miller N., Ye.Z, "Report on distributed generation penetration study", NREL, SR-560-34715,August 2003

[2] A.S. Anees, "Grid integration of renewable energy sources: Challenges, issues and possible solutions", 5th Inter. Conf. on Power Electronics (IICPE), 2012

[3] EN 50160, Voltage characteristics of electricity supplied by public distribution systems, 1999 
[4] H. Markiewicz, A. Klajn, "Voltage Disturbances, Standard EN 50160 - Voltage Characteristics in Public Distribution Systems", Leonardo Power Quality Initiative, July 2004

[5] T.A. Short, "Distribution Reliability and Power Quality", CRC Press, 2005

[6] L. Gelazanskas, K.A.A. Gamage, "Demand side management in smart grid: A review and proposals for future direction", Sustainable cities and society, Volume 11, February 2014

[7] K.S. Reddy, M. Kumar, T.K. Mallick, H.Saron, S. Lokeswaran, "A review of Integration, Control, Communication and Metering (ICCM) of renewable energy based smart grid", Renewable and Sustainable Energy Reviews, Vol. 38, October 2014

[8] E. S. Karapidakis, Y. A. Katsigiannis, P. S. Georgilakis, E. Thalassinakis, "Generation Expansion Planning of Crete Power System for High Penetration of Renewable Energy Sources", Materials Science Forum, Vol 670, pp. 407-414, Dec. 2010

[9] A.Tsikalakis, N. Hatziargyriou, K. Papadogiannis, A.Gigantidou, J. Stefanakis, E. Thalassinakis "Financial Contribution of Wind Power on the Island System of Crete", Proc of RES for islands conference, Crete, May 2003, pp 21-31.

[10] N.Hatziargyriou, A.Dimeas, A.Tsikalakis A. Gigantidou, E. Thalassinakis, "Intelligent Techniques applied to the Economic and Secure Operation of Island Systems with Large Wind Power Penetration". CD proc. of the 12th ISAP 03, Lemnos, September 2003, ISAP 03/162.

[11]REN 21, "Renewables 2014 Global Status Report." URL: http://www.ren21.net/.

[12] Antonios G. Tsikalakis, Nikolaos D. Hatziargyriou, Emmanuel Karapidakis, and Emmanuel Kymakis, "Economic evaluation of low Photovoltaics (PV) penetration in island power systems, application to Crete", proc of the DEMSEE conference, Sitia, Crete, Greece, 23-24 September 2010.

[13] CIGRE,"Benchmark Systems for Network Integration of Renewable and Distributed Energy Resources", technical brochure No575

[14]EN 50160, "Voltage characteristics of electricity supplied by public distribution systems", 1999

[15]IEA PVPS, “Impacts of Power Penetration from Photovoltaic Power Systems in Distribution Networks", Report T5-10, 2002
[16]B. Blazic, T. Pfajfar, I. Papic, "Voltage Control in Networks with Distributed Generation - A Case Study”, Sustainable Alternative Energy (SAE), 2009 IEEE PES/IAS Conference

[17] V.V. Thong, J.Driesen, R. Belmans, "Improving voltage stability with the connection of distributed generation", International conference on power and energy systems (IASTED) Marbella, Spain, 2003

[18] Th. Boutsika, S. Papathanassiou, N. Drossos, "Calculation of the Fault Level Contribution of Distributed Generation according to IEC Standard 60909". Proc. CIGRE Symposium "Power Systems with Dispersed Generation", Athens, April 2005

[19] "Technical Guideline Generating Plants Connected to the Medium-Voltage Network", BDEW, June 2008

[20] G. Chicco, J. Schlabbach, F. Spertino, “Experimental assessment of the waveform distortion in gridconnected photovoltaic installations", Solar Energy, 83,2009

[21] "Distributed generation with high penetration of Renewable Energy Sources", DISPOWER Project, Final Public Report, 2006

[22] Tomoki Ehara, "Overcoming PV grid issues in urban areas", Report IEA-PVPS T10-06-2009

[23] T.E.Hoff, R. Perez, "Modeling PV fleet output variability", Solar Energy, Vol 86, Issue 8, August 2012

[24] T.E.Hoff, R. Perez, “Quantifying PV power output variability”, Solar Energy, Vol 84, Issue 10, October 2010

[25]F. Mavromatakis, G. Makrides, G. Georghiou, A. Pothrakis, Y. Fraghiadakis, E. Drakakis, E. Koudoumas, "Modelling the photovoltaic potential of a site", Renewable Energy, 35, 1387-1390, 2010

[26]F. Mavromatakis, F. Vignola, Y. Fraghiadakis, "Modelling the power produced by photovoltaic systems“, ARC14, Qatar 2014. 